\title{
AN ENTROPY SATISFYING SCHEME FOR TWO-LAYER SHALLOW WATER EQUATIONS WITH UNCOUPLED TREATMENT
}

\author{
François Bouchut ${ }^{1}$ And Tomás Morales De LunA ${ }^{2}$
}

\begin{abstract}
We consider the system of partial differential equations governing the one-dimensional flow of two superposed immiscible layers of shallow water. The difficulty in this system comes from the coupling terms involving some derivatives of the unknowns that make the system nonconservative, and eventually nonhyperbolic. Due to these terms, a numerical scheme obtained by performing an arbitrary scheme to each layer, and using time-splitting or other similar techniques leads to instabilities in general. Here we use entropy inequalities in order to control the stability. We introduce a stable well-balanced time-splitting scheme for the two-layer shallow water system that satisfies a fully discrete entropy inequality. In contrast with Roe type solvers, it does not need the computation of eigenvalues, which is not simple for the two-layer shallow water system. The solver has the property to keep the water heights nonnegative, and to be able to treat vanishing values.
\end{abstract}

Mathematics Subject Classification. 74S10, 35L60, 74G15.

Received March 5, 2007. Revised December 11, 2007.

Published online June 5, 2008.

\section{THE TWO-LAYER SHALLOW WATER SYSTEM}

The two-layer shallow water system of partial differential equations governs the one-dimensional flow of two superposed shallow layers of immiscible fluids with different constant densities

$$
0<\rho_{1}<\rho_{2}
$$

over a bottom with topography $z(x)$. According to [10], it writes as

$$
\left\{\begin{array}{l}
\partial_{t} h_{1}+\partial_{x}\left(h_{1} u_{1}\right)=0 \\
\partial_{t}\left(h_{1} u_{1}\right)+\partial_{x}\left(h_{1} u_{1}^{2}+\frac{g}{2} h_{1}^{2}\right)=-g h_{1} \partial_{x} h_{2}-g h_{1} \partial_{x} z, \\
\partial_{t} h_{2}+\partial_{x}\left(h_{2} u_{2}\right)=0, \\
\partial_{t}\left(h_{2} u_{2}\right)+\partial_{x}\left(h_{2} u_{2}^{2}+\frac{g}{2} h_{2}^{2}\right)=-\frac{\rho_{1}}{\rho_{2}} g h_{2} \partial_{x} h_{1}-g h_{2} \partial_{x} z,
\end{array}\right.
$$

Keywords and phrases. Two-layer shallow water, nonconservative system, complex eigenvalues, time-splitting, entropy inequality, well-balanced scheme, nonnegativity.

${ }^{1}$ Département de Mathématiques et Applications, École Normale Supérieure \& CNRS, 45 rue d’Ulm, 75230 Paris cedex 05, France. francois.bouchut@ens.fr

2 Departamento de Anlálisis Matemático, Facultad de Ciencias, Universidad de Málaga, Campus de Teatinons s/n, 29071 Málaga, Spain. morales@anamat.cie.uma.es 


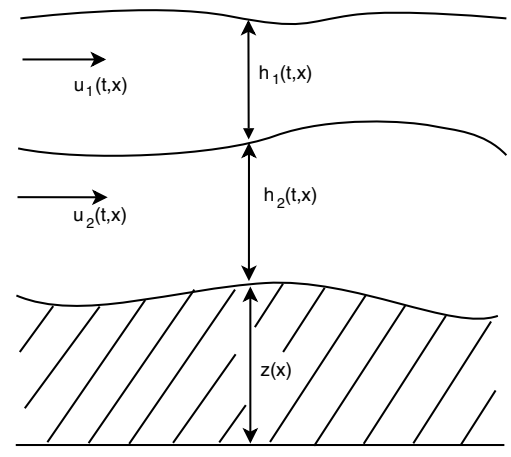

FiguRE 1. Two-layer shallow water model with topography.

where $u_{i}$ is the horizontal velocity of the $i$ th layer, $h_{i} \geq 0$ is the layer depth and $g$ is the gravity constant. In these equations, index 1 makes reference to the upper layer and index 2 to the lower one, see Figure 1.

The evolution of a single layer of shallow water can be classically described by the Saint Venant model with source term

$$
\left\{\begin{array}{l}
\partial_{t} h+\partial_{x}(h u)=0 \\
\partial_{t}(h u)+\partial_{x}\left(h u^{2}+\frac{g}{2} h^{2}\right)=-g h \partial_{x} z
\end{array}\right.
$$

As soon as the topography $z(x)$ is smooth, this system is a classical conservative hyperbolic system with source, with eigenvalues $u \pm \sqrt{g h}$. It has a convex entropy (the physical energy)

$$
\widehat{\eta}(U, z)=h u^{2} / 2+g h^{2} / 2+g h z,
$$

with entropy flux

$$
\widehat{G}(U, z)=\left(h u^{2} / 2+g h^{2}+g h z\right) u,
$$

where $U=(h, h u)$. Thus weak entropy solutions to (1.3) must satisfy

$$
\partial_{t} \widehat{\eta}(U, z)+\partial_{x} \widehat{G}(U, z) \leq 0 \text {. }
$$

\subsection{Difficulties in the two-layer system}

The main difference between the one-layer shallow water system (1.3) and the two-layer one (1.2) is that the latter is not a conservative system, because of the products $h_{1} \partial_{x} h_{2}$ and $h_{2} \partial_{x} h_{1}$ in the right-hand sides. Classically, such products are not well-defined for discontinuous solutions, and can lead to several notions of weak solutions. In practice, there is a lack of Rankine-Hugoniot condition in order to define jump relations at a discontinuity. Indeed, here, only one scalar relation is missing because the total momentum admits a conservative equation

$$
\partial_{t}\left(\rho_{1} h_{1} u_{1}+\rho_{2} h_{2} u_{2}\right)+\partial_{x}\left(\rho_{1}\left(h_{1} u_{1}^{2}+\frac{g}{2} h_{1}^{2}\right)+\rho_{2}\left(h_{2} u_{2}^{2}+\frac{g}{2} h_{2}^{2}\right)+\rho_{1} g h_{1} h_{2}\right)=-\left(\rho_{1} h_{1}+\rho_{2} h_{2}\right) g \partial_{x} z,
$$

giving a Rankine-Hugoniot relation (if $z$ is smooth or if the discontinuity is not steady). Several solutions can exist and several criteria of selection have already been proposed, see [12-14]. We refer to $[1,7,8]$ for some other examples. In this paper we shall not be able to impose any condition (except the entropy inequality, see below) in order to define weak solutions to the system so that the solutions may differ from the ones computed with other numerical schemes. Nevertheless, we consider any possible solution that satisfies an entropy inequality. 
A second difficulty comes from the complexity of the eigenstructure of (1.2). We shall denote the variable and the nonlinearity of (1.3) as

$$
U=(h, h u), \quad \widehat{F}(U)=\left(h u, h u^{2}+\frac{g}{2} h^{2}\right)
$$

and the variable and the conservative part of (1.2) as

$$
\widetilde{U}=\left(U_{1}, U_{2}\right)=\left(h_{1}, h_{1} u_{1}, h_{2}, h_{2} u_{2}\right), \quad F(\widetilde{U})=\left(\widehat{F}\left(U_{1}\right), \widehat{F}\left(U_{2}\right)\right) .
$$

Then, the system (1.2) can be written under the quasilinear form

$$
\partial_{t}\left(\begin{array}{c}
\widetilde{U} \\
z
\end{array}\right)+A(\widetilde{U}) \partial_{x}\left(\begin{array}{c}
\widetilde{U} \\
z
\end{array}\right)=0
$$

where

$$
A(\widetilde{U})=\left(\begin{array}{cc|cc|c}
\widehat{A}\left(U_{1}\right) & 0 & 0 & 0 \\
\hline 0 & 0 & \widehat{A}\left(U_{2}\right) & 0 & g h_{1} \\
\hline \frac{\rho_{1}}{\rho_{2}} g h_{2} & 0 & 0 & g h_{2} \\
\hline 0 & 0 & 0 & 0 & 0
\end{array}\right), \quad \widehat{A}(U)=\widehat{F}^{\prime}(U)=\left(\begin{array}{cc}
0 & 1 \\
-u^{2}+g h & 2 u
\end{array}\right) .
$$

The eigenvalues of the matrix $A$ cannot be explicitly calculated, and indeed they may become complex, losing the hyperbolicity of the system. This occurs in practice when the difference $u_{1}-u_{2}$ is large. This means that the linearization of the system around a constant state is ill-posed. However, it is believed that the elliptic region has a repulsive role, and if the solution approaches this region, it is rapidly evacuated to its boundary. So, even if complex eigenvalues exist, well-posedness is nevertheless expected for strong enough nonlinearity.

The use of the two-layer system even when complex eigenvalues occur has several motivations. First, if the solution stays only little on the elliptic region, one would like still to have a numerical result, the small region affecting only little the full solution. Second, this situation is related to Kelvin-Helmoltz instability and the physical behavior is related to mixing and turbulence. The resolution of the two-layer system in this situation provides a rough description to the complex flow that should arise instead.

In practice, when using a Roe type scheme, a numerical computation of the eigenvalues is necessary. This implies an additional computational cost, and moreover a special treatment of complex values.

Another important feature of system (1.2) is that the depths $h_{1}, h_{2}$ are nonnegative. It is important to preserve this property at the numerical level, and also to be able to treat vanishing values. This is not the case in Roe type schemes.

A good property of system (1.2) is that it admits a global convex entropy, the physical energy. Thus admissible weak solutions should satisfy the corresponding entropy inequality

$$
\partial_{t} \widetilde{\eta}(\widetilde{U}, z)+\partial_{x} \widetilde{G}(\widetilde{U}, z) \leq 0,
$$

where

$$
\begin{gathered}
\eta(\widetilde{U})=\rho_{1}\left(h_{1} \frac{u_{1}^{2}}{2}+g \frac{h_{1}^{2}}{2}\right)+\rho_{2}\left(h_{2} \frac{u_{2}^{2}}{2}+g \frac{h_{2}^{2}}{2}\right)+\rho_{1} g h_{1} h_{2}, \\
G(\widetilde{U})=\rho_{1}\left(h_{1} \frac{u_{1}^{2}}{2}+g h_{1}^{2}\right) u_{1}+\rho_{2}\left(h_{2} \frac{u_{2}^{2}}{2}+g h_{2}^{2}\right) u_{2}+\rho_{1} g h_{1} h_{2}\left(u_{1}+u_{2}\right), \\
\widetilde{\eta}(\widetilde{U}, z)=\eta(\widetilde{U})+\rho_{1} g h_{1} z+\rho_{2} g h_{2} z, \quad \widetilde{G}(\widetilde{U}, z)=G(\widetilde{U})+\rho_{1} g h_{1} u_{1} z+\rho_{2} g h_{2} u_{2} z .
\end{gathered}
$$


At the numerical level it is not obvious to satisfy a discrete entropy inequality. However, if this is the case, this ensures a kind of global nonlinear stability property, because of the decrease of the total entropy.

Taking into account the above properties, the leading idea of the present paper for solving (1.2) is to enforce a discrete entropy inequality by considering a time-splitting method. The advantage of our approach is that it avoids the computation of eigenvalues, and the problem of complex values. It also enables easily to preserve the nonnegativity of the layers depths. The discrete entropy inequality controls at the same time the admissibility of the solutions and the global nonlinear stability of the scheme.

\subsection{Steady states and well-balanced schemes}

In the one-layer shallow water system (1.3), the steady states at rest, where $u \equiv 0$ and $h+z=$ cst play an important role, since they can be considered as reference solutions. For the two-layer shallow water system (1.2), one can get the steady states by writing the equations satisfied by $u_{1}$ and $u_{2}$ (for smooth solutions),

$$
\begin{gathered}
\partial_{t} u_{1}+\partial_{x}\left(\frac{u_{1}^{2}}{2}+g\left(h_{1}+h_{2}+z\right)\right)=0, \\
\partial_{t} u_{2}+\partial_{x}\left(\frac{u_{2}^{2}}{2}+g\left(\frac{\rho_{1}}{\rho_{2}} h_{1}+h_{2}+z\right)\right)=0 .
\end{gathered}
$$

Therefore, the steady states $\widetilde{U}(x)$ are characterized by

$$
\left\{\begin{array}{l}
h_{1} u_{1}=\text { Cst } \\
\frac{u_{1}^{2}}{2}+g\left(h_{1}+h_{2}+z\right)=\text { Cst } \\
h_{2} u_{2}=\text { Cst, } \\
\frac{u_{2}^{2}}{2}+g\left(h_{2}+\frac{\rho_{1}}{\rho_{2}} h_{1}+z\right)=\text { Cst. }
\end{array}\right.
$$

Between them, the steady states at rest, for which $u_{i} \equiv 0$, are characterized by

$$
u_{1}=u_{2}=0, \quad h_{1}=\mathrm{cst}, \quad h_{2}+z=\text { cst. }
$$

When solving numerically (1.2), it is important to be able to preserve theses steady states at the discrete level in order to be able to accurately compute the evolution of small deviations from them. Indeed, the majority of real-life applications resides in this flow regime. The numerical schemes satisfying this property are called wellbalanced. Well-balanced schemes have been derived for the one-layer shallow water system by various authors, see in particular $[3-6,9,15,17]$. For the case of two layers, some schemes have been proposed in $[2,11,16]$.

\section{NumericAl METHOD}

The two-layer shallow water system (1.2) can be interpreted as two coupled Saint Venant systems (1.3) where the topography depends on the other layer height (the topography is $z+h_{2}$ for the first layer, and $z+\frac{\rho_{1}}{\rho_{2}} h_{1}$ for the second layer). This is not exactly true because these apparent topographies $z+h_{2}$ and $z+\frac{\rho_{1}}{\rho_{2}} h_{1}$ do depend on time. This nevertheless happens to be true when using a time-splitting method, as is explained below. Then the difficulty of nonconservative products disappears apparently. However, a numerical scheme obtained by performing an arbitrary scheme to each layer independently of the other leads to unstable schemes in general, see for example [11]. Our method does not show instabilities because it is compatible with the entropy inequality. Indeed we use a two-step algorithm so that in each step a Saint Venant problem is solved and the total entropy for the two-layer system is dissipated. 
The remainder of this section is organized as follows. We first recall in Section 2.1 how to write discrete entropy inequalities. Then, Section 2.2 explains a general setting for time-splitting methods, and Section 2.3 finally introduces our entropy satisfying method.

\subsection{Quasilinear systems and entropy satisfying schemes}

We recall that, given a quasilinear system

$$
\partial_{t} U+A(U) \partial_{x} U=0
$$

an entropy for the system is a convex function $\eta(U)$ such that there exists a function $G(U)$, called entropy flux, that verifies $\eta^{\prime} A=G^{\prime}$. A weak solution to (2.1) (in a sense to be specified) is said to be entropy satisfying relatively to $\eta$ if

$$
\partial_{t} \eta(U)+\partial_{x} G(U) \leq 0
$$

We consider a grid of points $x_{i+1 / 2}, i \in \mathbb{Z}$, and we define the discrete values $U_{i}^{n}$ which intend to be approximations of the averages of the exact solution over the cells $C_{i}=\left(x_{i-1 / 2}, x_{i+1 / 2}\right)$ at time $t_{n}=n \Delta t$,

$$
U_{i}^{n} \simeq \frac{1}{\triangle x_{i}} \int_{C_{i}} U\left(t_{n}, x\right) \mathrm{d} x
$$

where $\triangle x_{i}=x_{i+1 / 2}-x_{i-1 / 2}$. A first-order explicit three points scheme for the system (2.1) is a formula of the form

$$
U_{i}^{n+1}-U_{i}^{n}+\frac{\triangle t}{\triangle x_{i}}\left(F_{i+1 / 2-}-F_{i-1 / 2+}\right)=0
$$

where

$$
F_{i+1 / 2-}=\mathcal{F}_{l}\left(U_{i}, U_{i+1}\right), \quad F_{i+1 / 2+}=\mathcal{F}_{r}\left(U_{i}, U_{i+1}\right),
$$

and $\mathcal{F}_{l}, \mathcal{F}_{r}$ are numerical fluxes consistent in some sense with the system. We always assume that $\mathcal{F}_{l}(U, U)=$ $\mathcal{F}_{r}(U, U)$, and we refer for example to [9] for further details. Indeed, the consistency of the numerical fluxes should be defined according to the nature of the system (2.1).

In the case of the Saint Venant system (1.3), that can be written as a quasilinear system in the variable $(U, z)=(h, h u, z)$, the good notion of consistency for numerical fluxes $\widehat{\mathcal{F}}_{l}\left(U_{l}, U_{r}, z_{l}, z_{r}\right), \widehat{\mathcal{F}}_{r}\left(U_{l}, U_{r}, z_{l}, z_{r}\right)$ is as follows [9]. We first require the consistency with the conservative part

$$
\widehat{\mathcal{F}}_{l}(U, U, z, z)=\widehat{\mathcal{F}}_{r}(U, U, z, z)=\widehat{F}(U)=\left(h u, h u^{2}+\frac{g}{2} h^{2}\right) .
$$

Second, denoting the components of the numerical fluxes by $\widehat{\mathcal{F}}_{l}=\left(\widehat{\mathcal{F}}_{l}^{0}, \widehat{\mathcal{F}}_{l}^{1}\right), \widehat{\mathcal{F}}_{r}=\left(\widehat{\mathcal{F}}_{r}^{0}, \widehat{\mathcal{F}}_{r}^{1}\right)$, we require conservativity of water height, and consistency with the source/asymptotic conservativity of momentum

$$
\begin{gathered}
\widehat{\mathcal{F}}_{l}^{0}\left(U_{l}, U_{r}, z_{l}, z_{r}\right)=\widehat{\mathcal{F}}_{r}^{0}\left(U_{l}, U_{r}, z_{l}, z_{r}\right) \equiv \widehat{\mathcal{F}}^{0}\left(U_{l}, U_{r}, z_{l}, z_{r}\right), \\
\widehat{\mathcal{F}}_{r}^{1}\left(U_{l}, U_{r}, z_{l}, z_{r}\right)-\widehat{\mathcal{F}}_{l}^{1}\left(U_{l}, U_{r}, z_{l}, z_{r}\right)=-g h\left(z_{r}-z_{l}\right)+o\left(z_{r}-z_{l}\right),
\end{gathered}
$$

as $U_{l}, U_{r} \rightarrow U$ and $z_{l}, z_{r} \rightarrow z$.

The stability of the scheme can be controlled via a discrete entropy inequality, which is a discrete version of the inequality (2.2). We have the following two definitions.

Definition 2.1. We say that the scheme (2.4) satisfies a discrete entropy inequality associated to the convex entropy $\eta$ for (2.1), if there exists a numerical entropy flux function $\mathcal{G}\left(U_{l}, U_{r}\right)$ which is consistent with the exact 
entropy flux (in the sense that $\mathcal{G}(U, U)=G(U)$ ), such that, under some CFL condition, the discrete values computed by (2.4) automatically satisfy

$$
\eta\left(U_{i}^{n+1}\right)-\eta\left(U_{i}^{n}\right)+\frac{\triangle t}{\triangle x_{i}}\left(G_{i+1 / 2}-G_{i-1 / 2}\right) \leq 0,
$$

with

$$
G_{i+1 / 2}=\mathcal{G}\left(U_{i}^{n}, U_{i+1}^{n}\right)
$$

Definition 2.2. We say that the scheme (2.4) satisfies a semi-discrete entropy inequality associated to the convex entropy $\eta$ for $(2.1)$, if there exists a numerical entropy flux function $\mathcal{G}\left(U_{l}, U_{r}\right)$ which is consistent with the exact entropy flux (in the sense that $\mathcal{G}(U, U)=G(U)$ ), such that for any $U_{l}, U_{r}$,

$$
\begin{aligned}
& G\left(U_{r}\right)+\eta^{\prime}\left(U_{r}\right)\left(\mathcal{F}_{r}\left(U_{l}, U_{r}\right)-\mathcal{F}_{r}\left(U_{r}, U_{r}\right)\right) \leq \mathcal{G}\left(U_{l}, U_{r}\right), \\
& \mathcal{G}\left(U_{l}, U_{r}\right) \leq G\left(U_{l}\right)+\eta^{\prime}\left(U_{l}\right)\left(\mathcal{F}_{l}\left(U_{l}, U_{r}\right)-\mathcal{F}_{l}\left(U_{l}, U_{l}\right)\right) .
\end{aligned}
$$

This definition is indeed equivalent to the inequality (2.9) in the limit $\Delta t \rightarrow 0$. For further details, we refer again to [9].

\subsection{Time-splitting schemes for quasilinear systems}

A time-splitting technique leads to a two-step scheme. Consider the system

$$
\partial_{t} U+(\mathcal{A}(U)+\mathcal{B}(U)) \partial_{x} U=0
$$

The idea of the time-splitting technique is that starting from a function $U^{n}(x)$ at time $t_{n}$, instead of solving (2.12) over $t \in\left(t_{n}, t_{n+1}\right)$ with initial data $U^{n}(x)$, we rather first solve the system

$$
\left\{\begin{array}{l}
\partial_{t} U^{1}+\mathcal{A}\left(U^{1}\right) \partial_{x} U^{1}=0, \quad t \in\left(t_{n}, t_{n+1}\right), \\
U^{1}\left(t_{n}, x\right)=U^{n}(x) .
\end{array}\right.
$$

Then we solve the system

$$
\left\{\begin{array}{l}
\partial_{t} U^{2}+\mathcal{B}\left(U^{2}\right) \partial_{x} U^{2}=0, \\
U^{2}\left(t_{n}, x\right)=U^{1}\left(t_{n+1}, x\right) .
\end{array} \quad t \in\left(t_{n}, t_{n+1}\right),\right.
$$

Finally, we take for approximate value at time $t_{n+1}$ the function $U^{n+1}(x)=U^{2}\left(t_{n+1}, x\right)$.

At the discrete level, we consider as above a grid of points $x_{i+1 / 2}, i \in \mathbb{Z}$, and the cells $C_{i}=\left(x_{i-1 / 2}, x_{i+1 / 2}\right)$. The approximations $U_{i}^{n}$ of the averages of the solution to (2.12) are then updated in two steps which correspond to discrete schemes for (2.13) and (2.14).

Definition 2.3. A time-splitting scheme for (2.12) is a formula

$$
\begin{aligned}
& U_{i}^{n+1 / 2}=U_{i}^{n}-\frac{\triangle t}{\triangle x_{i}}\left(\mathcal{F}_{l}^{1}\left(U_{i}^{n}, U_{i+1}^{n}\right)-\mathcal{F}_{r}^{1}\left(U_{i-1}^{n}, U_{i}^{n}\right)\right), \\
& U_{i}^{n+1}=U_{i}^{n+1 / 2}-\frac{\triangle t}{\triangle x_{i}}\left(\mathcal{F}_{l}^{2}\left(U_{i}^{n+1 / 2}, U_{i+1}^{n+1 / 2}\right)-\mathcal{F}_{r}^{2}\left(U_{i-1}^{n+1 / 2}, U_{i}^{n+1 / 2}\right)\right),
\end{aligned}
$$

where $\mathcal{F}_{l}^{1}, \mathcal{F}_{r}^{1}$ are consistent numerical fluxes for the system $(2.13)$ and $\mathcal{F}_{l}^{2}, \mathcal{F}_{r}^{2}$ are consistent numerical fluxes for the system (2.14).

Consider now an entropy $\eta$ for the system (2.12), that is, $\eta$ is a scalar convex function of $U$ such that there exists an entropy flux $G$ that verifies $\eta^{\prime}(\mathcal{A}+\mathcal{B})=G^{\prime}$. 
Definition 2.4. We say that the time-splitting scheme (2.15) is entropy compatible with respect to the entropy $\eta$ of (2.12) if:

(i) The function $\eta$ is an entropy for both systems (2.13) and (2.14). In other words, there must exist some entropy fluxes $G^{1}, G^{2}$ such that

$$
\eta^{\prime} \mathcal{A}=\left(G^{1}\right)^{\prime}, \quad \eta^{\prime} \mathcal{B}=\left(G^{2}\right)^{\prime} .
$$

(ii) Each of the two schemes in (2.15) satisfies a discrete entropy inequality associated to $\eta$. In other words, there exist two numerical entropy fluxes $\mathcal{G}^{1}\left(U_{l}, U_{r}\right), \mathcal{G}^{2}\left(U_{l}, U_{r}\right)$ consistent with the exact entropy fluxes $\left(\mathcal{G}^{1}(U, U)=\right.$ $\left.G^{1}(U), \mathcal{G}^{2}(U, U)=G^{2}(U)\right)$, such that under some CFL conditions, the discrete values computed by (2.15) satisfy

$$
\begin{gathered}
\eta\left(U_{i}^{n+1 / 2}\right)-\eta\left(U_{i}^{n}\right)+\frac{\triangle t}{\triangle x_{i}}\left(G_{i+1 / 2}^{1}-G_{i-1 / 2}^{1}\right) \leq 0, \\
\eta\left(U_{i}^{n+1}\right)-\eta\left(U_{i}^{n+1 / 2}\right)+\frac{\triangle t}{\triangle x_{i}}\left(G_{i+1 / 2}^{2}-G_{i-1 / 2}^{2}\right) \leq 0,
\end{gathered}
$$

with $G_{i+1 / 2}^{1}=\mathcal{G}^{1}\left(U_{i}^{n}, U_{i+1}^{n}\right)$, and $G_{i+1 / 2}^{2}=\mathcal{G}^{2}\left(U_{i}^{n+1 / 2}, U_{i+1}^{n+1 / 2}\right)$.

The conditions (2.16) imply that up to a constant, one has $G=G^{1}+G^{2}$. Adding the two inequalities in (2.17) gives

$$
\eta\left(U_{i}^{n+1}\right)-\eta\left(U_{i}^{n}\right)+\frac{\triangle t}{\triangle x_{i}}\left(G_{i+1 / 2}-G_{i-1 / 2}\right) \leq 0
$$

with

$$
G_{i+1 / 2}=G_{i+1 / 2}^{1}+G_{i+1 / 2}^{2}=\mathcal{G}^{1}\left(U_{i}^{n}, U_{i+1}^{n}\right)+\mathcal{G}^{2}\left(U_{i}^{n+1 / 2}, U_{i+1}^{n+1 / 2}\right) .
$$

Thus it generalizes Definition 2.1.

Definition 2.5. We say that the time-splitting scheme (2.15) is semi-discrete entropy compatible with respect to the entropy $\eta$ of (2.12) if:

(i) The function $\eta$ is an entropy for both systems (2.13) and (2.14). In other words, there must exist some entropy fluxes $G^{1}, G^{2}$ such that

$$
\eta^{\prime} \mathcal{A}=\left(G^{1}\right)^{\prime}, \quad \eta^{\prime} \mathcal{B}=\left(G^{2}\right)^{\prime} .
$$

(ii) Each of the two schemes in (2.15) satisfies a semi-discrete entropy inequality associated to $\eta$. In other words, there exist two numerical entropy fluxes $\mathcal{G}^{1}\left(U_{l}, U_{r}\right), \mathcal{G}^{2}\left(U_{l}, U_{r}\right)$ consistent with the exact entropy fluxes $\left(\mathcal{G}^{1}(U, U)=G^{1}(U), \mathcal{G}^{2}(U, U)=G^{2}(U)\right)$, such that for any $U_{l}, U_{r}$,

$$
\begin{aligned}
& G^{\alpha}\left(U_{r}\right)+\eta^{\prime}\left(U_{r}\right)\left(\mathcal{F}_{r}^{\alpha}\left(U_{l}, U_{r}\right)-\mathcal{F}_{r}^{\alpha}\left(U_{r}, U_{r}\right)\right) \leq \mathcal{G}^{\alpha}\left(U_{l}, U_{r}\right), \\
& \mathcal{G}^{\alpha}\left(U_{l}, U_{r}\right) \leq G^{\alpha}\left(U_{l}\right)+\eta^{\prime}\left(U_{l}\right)\left(\mathcal{F}_{l}^{\alpha}\left(U_{l}, U_{r}\right)-\mathcal{F}_{l}^{\alpha}\left(U_{l}, U_{l}\right)\right),
\end{aligned} \quad \alpha=1,2 .
$$

This is a generalization of Definition 2.2. Indeed, (2.21) corresponds to the semi-discrete limit $\triangle t \rightarrow 0$ of $(2.17)$

\subsection{A time-splitting entropy scheme for the two-layer system}

In the particular case of a two-layer shallow water system, we decompose (1.10)-(1.11) in

$$
\partial_{t}\left(\begin{array}{c}
\widetilde{U} \\
z
\end{array}\right)+(\mathcal{A}(\widetilde{U})+\mathcal{B}(\widetilde{U})) \partial_{x}\left(\begin{array}{c}
\widetilde{U} \\
z
\end{array}\right)=0
$$

where

$$
\mathcal{A}(\widetilde{U})=\left(\begin{array}{c|cc|c}
\widehat{A}\left(U_{1}\right) & 0 & 0 & 0 \\
& g h_{1} & 0 & g h_{1} \\
\hline 0
\end{array}\right),
$$




$$
\mathcal{B}(\widetilde{U})=\left(\begin{array}{cc|c|c|}
\multicolumn{4}{|c|}{0} \\
\hline 0 & 0 & \widehat{A}\left(U_{2}\right) & 0 \\
\frac{\rho_{1}}{\rho_{2}} g h_{2} & 0 & & g h_{2} \\
\hline \multicolumn{4}{|c}{0}
\end{array} .\right.
$$

Thus, (2.13) reduces to a Saint Venant system (1.3) in the variable $U_{1}$ with bottom $z+h_{2}$, because during this first step one has $\partial_{t} U_{2}=0$. Similarly, (2.14) reduces to a Saint Venant system in the variable $U_{2}$ with bottom $z+\frac{\rho_{1}}{\rho_{2}} h_{1}$, because during this second step one has $\partial_{t} U_{1}=0$.

Consider now any numerical fluxes $\widehat{\mathcal{F}}_{l}\left(U_{l}, U_{r}, z_{l}, z_{r}\right), \widehat{\mathcal{F}}_{r}\left(U_{l}, U_{r}, z_{l}, z_{r}\right)$ for the Saint Venant system (1.3). According to Definition 2.3 and using the above decomposition, we get a time-splitting scheme for (1.2). But since $\widetilde{U}_{i}^{n+1 / 2}=\left(U_{1, i}^{n+1}, U_{2, i}^{n}\right)$, it takes the form

$$
\begin{gathered}
U_{1, i}^{n+1}=U_{1, i}^{n}-\frac{\triangle t}{\triangle x_{i}}\left(\widehat{\mathcal{F}}_{l}\left(U_{1, i}^{n}, U_{1, i+1}^{n}, z_{i}+h_{2, i}^{n}, z_{i+1}+h_{2, i+1}^{n}\right)\right. \\
\left.-\widehat{\mathcal{F}}_{r}\left(U_{1, i-1}^{n}, U_{1, i}^{n}, z_{i-1}+h_{2, i-1}^{n}, z_{i}+h_{2, i}^{n}\right)\right), \\
U_{2, i}^{n+1}=U_{2, i}^{n}-\frac{\triangle t}{\triangle x_{i}}\left(\widehat{\mathcal{F}}_{l}\left(U_{2, i}^{n}, U_{2, i+1}^{n}, z_{i}+\frac{\rho_{1}}{\rho_{2}} h_{1, i}^{n+1}, z_{i+1}+\frac{\rho_{1}}{\rho_{2}} h_{1, i+1}^{n+1}\right)\right. \\
\left.-\widehat{\mathcal{F}}_{r}\left(U_{2, i-1}^{n}, U_{2, i}^{n}, z_{i-1}+\frac{\rho_{1}}{\rho_{2}} h_{1, i-1}^{n+1}, z_{i}+\frac{\rho_{1}}{\rho_{2}} h_{1, i}^{n+1}\right)\right) .
\end{gathered}
$$

Proposition 2.6. Consider left and right numerical fluxes $\widehat{\mathcal{F}}_{l}\left(U_{l}, U_{r}, z_{l}, z_{r}\right), \widehat{\mathcal{F}}_{r}\left(U_{l}, U_{r}, z_{l}, z_{r}\right)$ for the Saint Venant problem with bottom (1.3) that satisfy a discrete (resp. semi-discrete) entropy inequality corresponding to $\widehat{\eta}$ in (1.4). Then, the time-slitting scheme (2.25)-(2.26) is discrete (resp. semi-discrete) entropy compatible with respect to the entropy $\widetilde{\eta}$ of (1.15).

Proof. The entropy $\widetilde{\eta}$ and entropy flux $\widetilde{G}$ of (1.15) for the two-layer shallow water system are related to the entropy $\widehat{\eta}$ in (1.4) and entropy flux $\widehat{G}$ in (1.5) corresponding to the Saint Venant system (1.3), via the identities

$$
\begin{gathered}
\widetilde{\eta}(\widetilde{U}, z)=\rho_{1} \widehat{\eta}\left(U_{1}, z\right)+\rho_{2} \widehat{\eta}\left(U_{2}, z\right)+\rho_{1} g h_{1} h_{2}, \\
\widetilde{G}(\widetilde{U}, z)=\rho_{1} \widehat{G}\left(U_{1}, z\right)+\rho_{2} \widehat{G}\left(U_{2}, z\right)+\rho_{1} g h_{1} h_{2}\left(u_{1}+u_{2}\right) .
\end{gathered}
$$

We remark that

and therefore

$$
\widehat{\eta}(U, z+\bar{z})=\widehat{\eta}(U, z)+g h \bar{z}, \quad \widehat{G}(U, z+\bar{z})=\widehat{G}(U, z)+g h u \bar{z},
$$

$$
\begin{gathered}
\widetilde{\eta}(\widetilde{U}, z)=\rho_{1} \widehat{\eta}\left(U_{1}, z+h_{2}\right)+\rho_{2} \widehat{\eta}\left(U_{2}, z\right)=\rho_{1} \widehat{\eta}\left(U_{1}, z\right)+\rho_{2} \widehat{\eta}\left(U_{2}, z+\frac{\rho_{1}}{\rho_{2}} h_{1}\right), \\
\widetilde{G}(\widetilde{U}, z)=\rho_{1} \widehat{G}\left(U_{1}, z+h_{2}\right)+\rho_{2} \widehat{G}\left(U_{2}, z+\frac{\rho_{1}}{\rho_{2}} h_{1}\right) .
\end{gathered}
$$

We have to prove the property (i), i.e. that $\widetilde{\eta}(\widetilde{U}, z)$ is an entropy for both systems with matrices $\mathcal{A}$ and $\mathcal{B}$.

Consider first the system with matrix $\mathcal{A}$. This is just the Saint Venant system on $U_{1}$ with bottom $z+h_{2}$, together with $\partial_{t} U_{2}=0$. Thus $\widehat{\eta}\left(U_{1}, z+h_{2}\right)$ is an entropy for this system, with entropy flux $\widehat{G}\left(U_{1}, z+h_{2}\right)$. From the first decomposition of $\widetilde{\eta}$ in $(2.29)$ and since $\partial_{t}\left(\widehat{\eta}\left(U_{2}, z\right)\right)=0$, we deduce that $\widetilde{\eta}$ is an entropy for the first system with matrix $\mathcal{A}$, with entropy flux

$$
\widetilde{G}^{1}(\widetilde{U}, z)=\rho_{1} \widehat{G}\left(U_{1}, z+h_{2}\right) .
$$

Similarly, the system with matrix $\mathcal{B}$ is just the Saint Venant system on $U_{2}$ with bottom $z+\frac{\rho_{1}}{\rho_{2}} h_{1}$, together with $\partial_{t} U_{1}=0$. From the second decomposition of $\widetilde{\eta}$ in (2.29) we deduce that $\widetilde{\eta}$ is an entropy for the second system with matrix $\mathcal{B}$, with entropy flux

$$
\widetilde{G}^{2}(\widetilde{U}, z)=\rho_{2} \widehat{G}\left(U_{2}, z+\frac{\rho_{1}}{\rho_{2}} h_{1}\right) .
$$

This proves (i), and property (ii) in the discrete or semi-discrete case follows obviously. 
Theorem 2.7. Consider left and right numerical fluxes $\widehat{\mathcal{F}}_{l}\left(U_{l}, U_{r}, z_{l}, z_{r}\right), \widehat{\mathcal{F}}_{r}\left(U_{l}, U_{r}, z_{l}, z_{r}\right)$ for the Saint Venant problem with bottom (1.3) that are consistent in the sense of (2.6)-(2.8), preserve nonnegativity of the water height $h$, satisfy a discrete (resp. semi-discrete) entropy inequality corresponding to $\widehat{\eta}$ in (1.4), and preserve the steady states at rest for the Saint Venant system. Then the time-splitting scheme defined by (2.25)-(2.26)

(i) preserves the nonnegativity of the layers depths $h_{i}, i=1,2$, and each depth is conservative;

(ii) preserves the steady-states at rest for the two-layer shallow water system;

(iii) satisfies a discrete (resp. semi-discrete) entropy inequality associated to the entropy $\widetilde{\eta}$;

(iv) is consistent with the two-layer system.

Proof. As before, the first step (2.25) can be interpreted as the solution to a Saint Venant problem in the variables $\left(h_{1}, h_{1} u_{1}\right)$ with bottom $z+h_{2}$, while $h_{2}, u_{2}$ remain unchanged. The second step (2.26) can be interpreted as the solution to a Saint Venant problem in the variables $\left(h_{2}, h_{2} u_{2}\right)$ with bottom $z+\frac{\rho_{1}}{\rho_{2}} h_{1}$, while $h_{1}$, $u_{1}$ remain unchanged.

With this interpretation, (i) is obvious since in each step the nonnegativity of each layer depth is preserved, and each depth evolves according to a conservative equation.

The property (ii) is neither difficult, since the steady-states relations (1.18) can be interpreted as the conjunction of the steady states relations for each Saint Venant system. The case of steady-states at rest $u_{i}=0$ is a sub-case of this property, and steady-states at rest are preserved in each step.

The property (iii) follows from Proposition 2.6.

Finally, for property (iv), we need to specify that here consistency refers to smooth solutions only. The result follows from the consistency of $\widehat{\mathcal{F}}_{l}, \widehat{\mathcal{F}}_{r}(2.6)-(2.8)$, and the techniques developed in [9]. We refer in particular to Proposition 4.3, p. 72, there, and we skip the proof.

A very interesting property of our time-splitting method is that, as is seen on the update formulas (2.25)(2.26), it does not involve any computation of the eigenvalues of the matrix (1.11) of the system. The consequences are that:

- the computational cost is rather low compared to Roe type methods;

- the CFL condition necessary for nonnegativity or for discrete entropy inequalities refers only to the eigenvalues of each Saint Venant system;

- eventual occurrence of loss of hyperbolicity of the system does not affect the scheme and Theorem 2.7 remains valid;

- it is straightforward to apply the method to the multi-layer shallow water system with an arbitrary number of layers.

However, one property is missing within our method: the asymptotic conservativity of the total momentum. This would mean in particular that the total momentum becomes conservative in the case when $z=$ cst, according to (1.7). Therefore, at this time, we are not able to prove that the corresponding Rankine-Hugoniot relation holds for a discontinuous numerical solution.

\section{Numerical tests}

This section is devoted to numerical tests using our time-splitting method introduced in Section 2.3. In general, a numerical scheme applied to each layer independently may lead to instabilities. For example, in [11] it is shown that a Q-scheme with uncoupled upwinding leads to an unconditionally unstable scheme. For our scheme here, the existence of an entropy inequality allows somehow to control these instabilities. However, this could be called nonlinear stability since the entropy controls the size of the computed solution, but not the size of a deviation from a reference solution for example (this could be called linear stability). This is true in particular when complex eigenvalues occur, since in this case the loss of hyperbolicity means linear instability, while our scheme stills computes bounded values in this situation (at least in the case of a fully discrete entropy inequality). 
Apart from the previous remark, a major issue in our method is indeed the choice of the solver used for the single-layer Saint Venant problem. We have tested two solvers. The first is the hydrostatic reconstruction solver of [5], which satisfies only a semi-discrete entropy inequality, and the second is the kinetic solver of [17], which satisfies a fully discrete entropy inequality. We consider several sets of initial data.

\section{Test 1}

The first set of initial data is one of the examples proposed in [11]. We take $g=9.81, \rho_{1} / \rho_{2}=0.98, z=0$, and

$$
\begin{aligned}
& h_{1}(t=0, x)= \begin{cases}0.5 & \text { if } x<0.5, \\
0.55 & \text { if } x>0.5,\end{cases} \\
& h_{2}(t=0, x)= \begin{cases}0.5, & \text { if } x<0.5, \\
0.45, & \text { if } x>0.5,\end{cases} \\
& u_{1}(t=0, x)=u_{2}(t=0, x)=2.5 .
\end{aligned}
$$

We solve the system for $x \in[0,1]$ with 100 cells, at CFL 0.5 . We use the hydrostatic reconstruction solver for the definition of the fluxes $\widehat{\mathcal{F}}_{l}, \widehat{\mathcal{F}}_{r}$ (with the Suliciu relaxation solver as associated homogeneous solver). Figure 2 shows the free surface and the interface between the two layers at time $t=0.05$ together with a zoom of the interface. As we see, there is no instability like described in [11], even-though some small oscillations arise at the interface. These oscillations are slightly reduced if we use a second-order reconstruction technique in space and time (as described in [9]), and diminish the CFL number. They do not produce instabilities for larger times. The existence of these oscillations is related to the fact that the entropy inequality is only semi-discrete, which explains the better behavior of the algorithm for smaller CFL number.

Next, we replace the previous Saint Venant fluxes $\widehat{\mathcal{F}}_{l}, \widehat{\mathcal{F}}_{r}$ by those of the kinetic solver of [17]. The advantage is that these fluxes verify a fully discrete entropy inequality. According to Proposition 2.6, this implies that we have a fully discrete entropy inequality for our two-layer scheme. The numerical result corresponding to the same test case run with the kinetic solver is shown in Figure 3. We observe that with the kinetic solver, our time-splitting method gives good results without the necessity of reducing the CFL number. Still, we remark some small oscillations at the interface in Figure 3 (notice that this is a zoom, thus they are indeed very small). It has been observed that they are reduced when $\rho_{1} / \rho_{2}$ is not close to 1 and they increase for $\rho_{1} / \rho_{2} \rightarrow 1$.

\section{Test 2}

The second set of initial data is $g=9.81, \rho_{1} / \rho_{2}=0.7, z=0$, and

$$
\begin{aligned}
& h_{2}(t=0, x)= \begin{cases}0.2 & \text { if } x<5, \\
1.8 & \text { if } x>5,\end{cases} \\
& h_{1}(t=0, x)+h_{2}(t=0, x)=2, \\
& u_{1}(t=0, x)=u_{2}(t=0, x)=0 .
\end{aligned}
$$

We take 500 points in the interval $[0,10]$, and use CFL 0.5 . We use first the hydrostatic solver. The result is shown in Figure 4 for time $t=1$. We remark a shock at the interface that does not look natural. Actually, this shock disappears when we use a second-order reconstruction technique in space and time and reduce the CFL condition, as we see in Figure 5. This shock may be generated by some weak instability near the original discontinuity at the interface. As $\Delta t \rightarrow 0$, the effect of this instability is reduced and the shock disappears.

We conclude that although we have a semi-discrete entropy inequality, some instabilities may arise in practice unless the CFL condition is sufficiently small, which is not satisfactory in practice. 


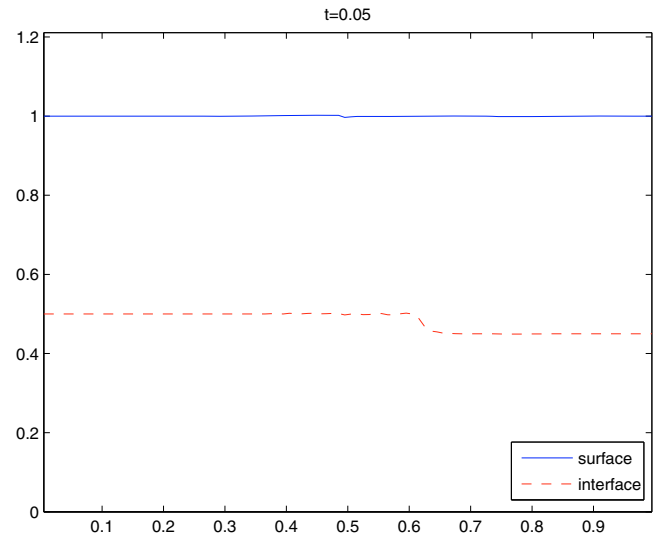

(a)

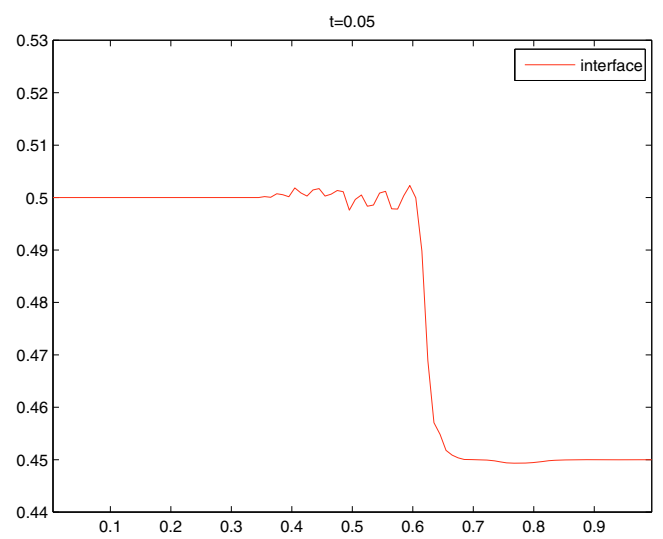

(b)

Figure 2. Test 1 with hydrostatic solver (first-order).

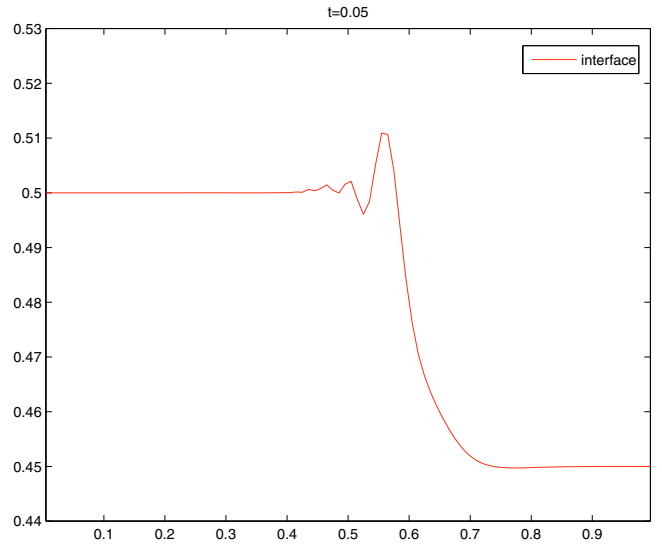

(a) First-order

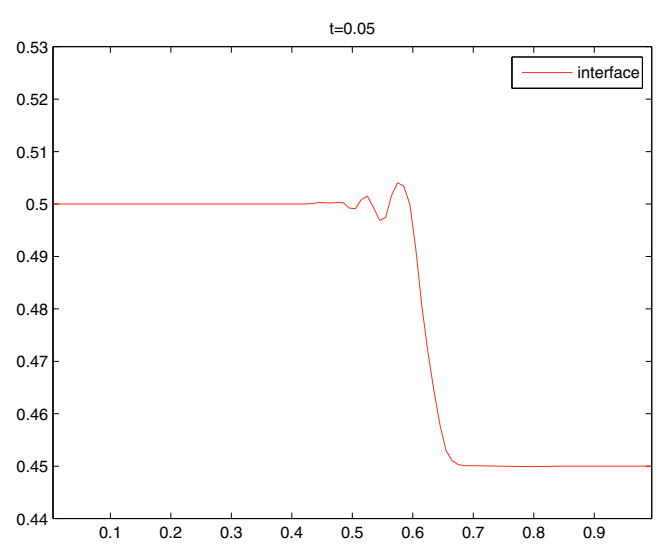

(b) Second-order

Figure 3. Test 1 with kinetic solver.

Next, we run the same test, but with the kinetic solver. The numerical result is shown in Figure 6 . We observe that the wrong shock disappears, without the necessity of reducing the CFL number.

However, when taking values of $\rho_{1} / \rho_{2}$ close to 1 , we observe that we can obtain a stationary shock in the interface, as we see in Figure 7. In this case, the shock does not disappear even if second-order is used. This shock may not be present when using other numerical schemes. In particular, according to M. Castro, the scheme developed in [15] does not show this shock, and the profile of the solution is then similar to the one given for $\rho_{1} / \rho_{2}=0.7$. We conclude that the weak solutions obtained by our time-splitting scheme may differ from the ones obtained with other schemes in the case $\rho_{1} / \rho_{2} \rightarrow 1$. 


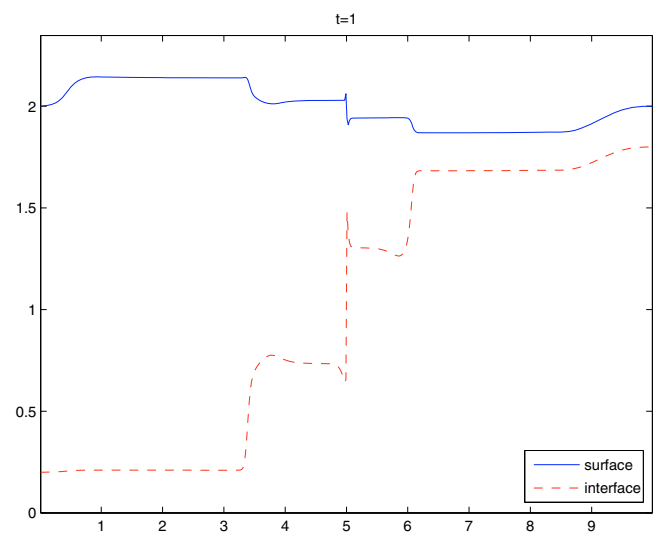

Figure 4. Test 2 with hydrostatic solver and CFL 0.5.

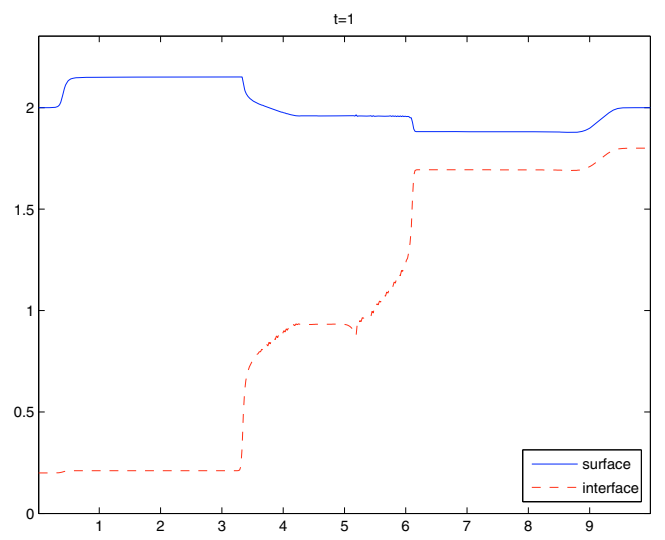

Figure 5. Test 2 with second-order hydrostatic solver and CFL 0.01.

\section{Test 3}

The third set of initial data is $g=9.81, \rho_{1} / \rho_{2}=0.98, z=0$, and

$$
\begin{aligned}
& h_{2}(t=0, x)= \begin{cases}0.5+0.01\left(1+\cos \left(\frac{(x-0.5) \pi}{0.1}\right)\right) & \text { if }|x-5|<0.1, \\
0.5 & \text { if }|x-5|>0.1\end{cases} \\
& h_{1}(t=0, x)+h_{2}(t=0, x)=1 \\
& u_{1}(t=0, x)=0.6 \\
& u_{2}(t=0, x)=-0.6
\end{aligned}
$$

According to the first-order approximations given in [18] for the eigenvalues of the two-layer system, we are in the case where the eigenvalues become complex, and the hyperbolicity is lost.

We solve the system for $x \in[0,10]$ with 1000 cells, at CFL 0.5. We use the kinetic solver. 


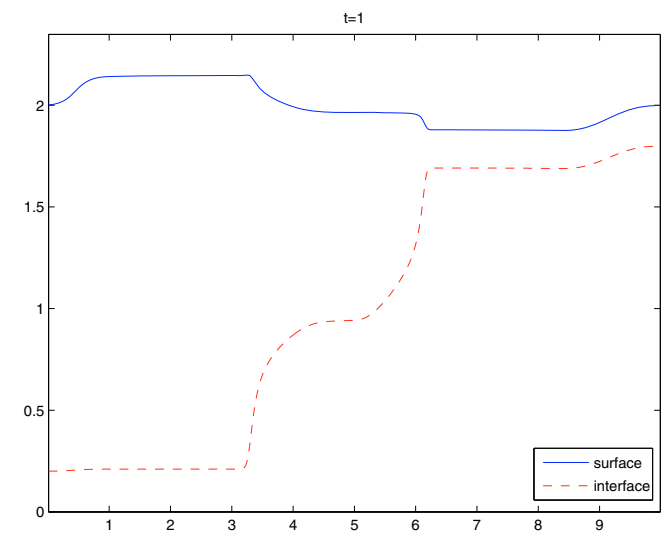

Figure 6. Test 2 with kinetic solver, first-order, CFL 0.5.

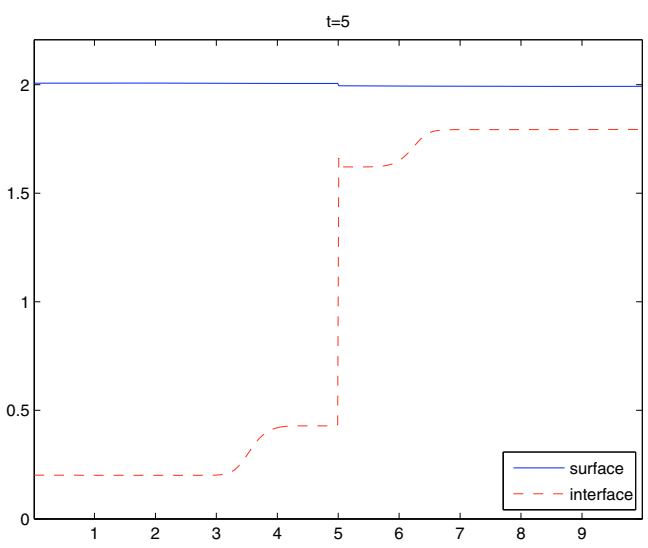

Figure 7. Test 2 for $\rho_{1} / \rho_{2}=0.98$ with kinetic solver, first-order, CFL 0.5.

This particular example does not make physical sense as the eigenvalues are complex in a large region and for a long period of time, but it shows that the scheme will not break down when we eventually find complex eigenvalues in other examples.

As we said before, our scheme is nonlinearly stable and it computes bounded values, as it is shown in Figure 8 for time $t=1$.

Even if the scheme does not break down, we can easily trace this occurrence of complex eigenvalues from the large number of oscillations shown at the interface, since the failure of hyperbolicity means linear instability.

\section{Test 4}

In the next example we take as initial data $g=9.81, z=0$, and

$$
\begin{aligned}
& h_{2}(t=0, x)= \begin{cases}1 & \text { if } x<0.5, \\
0 & \text { if } x>0.5,\end{cases} \\
& h_{1}(t=0, x)= \begin{cases}0 & \text { if } x<0.5, \\
1 & \text { if } x>0.5,\end{cases} \\
& u_{1}(t=0, x)=u_{2}(t=0, x)=0 .
\end{aligned}
$$




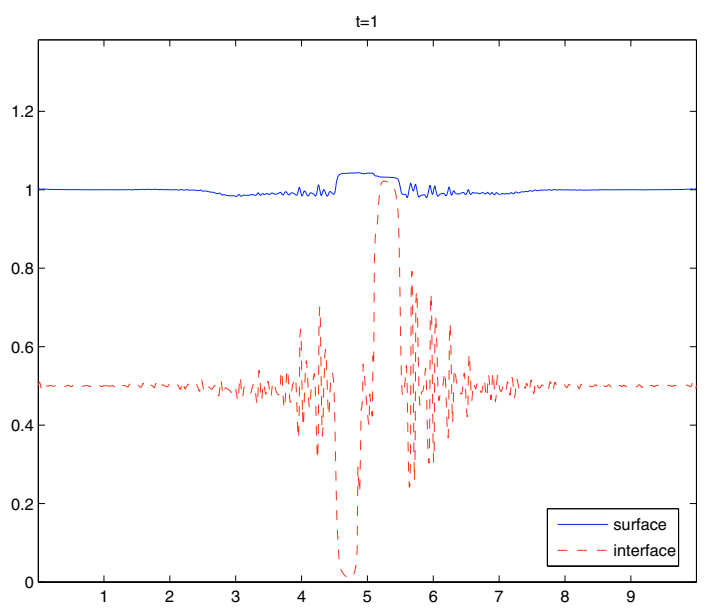

Figure 8. Test 3 with kinetic solver and CFL 0.5 .

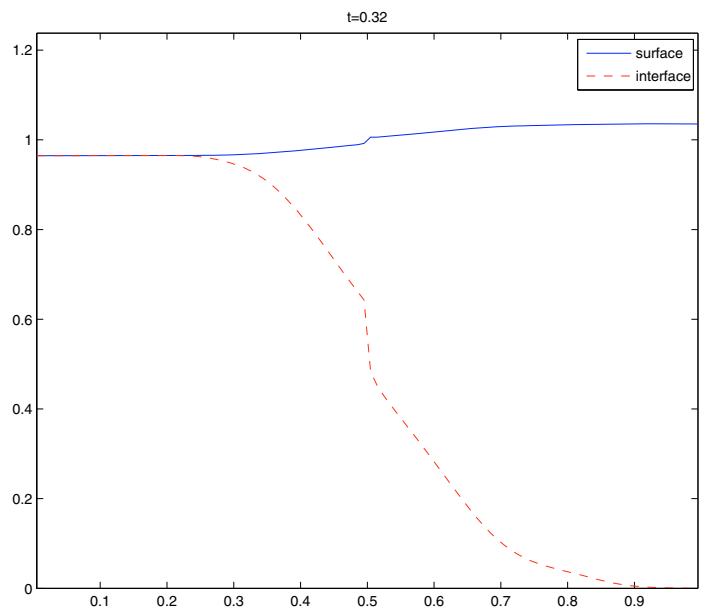

Figure 9. Test 4 with $\rho_{1} / \rho_{2}=0.85$ and kinetic solver.

We solve the system for $x \in[0,1]$ with 100 cells, at CFL 0.5 , using the kinetic solver and with Neumann boundary conditions. the results are shown in Figures 9 and 10 for $\rho_{1} / \rho_{2}=0.85$ and $\rho_{1} / \rho_{2}=0.95$ respectively.

As we see, void regions are treated correctly and the computed height of each layer is always nonnegative. In Figure 9 we observe a small steep region in the interface. It decreases and disappears for further time steps. On the contrary, in Figure 10 we get a stationary discontinuity in the interface that remains. We remark that the first-order approximations given in [18] for the eigenvalues become complex in some regions at certain times. Thus the discontinuity could be attached to complex eigenvalues. Even if the interpretation of the solution is unclear, the numerical solution given by the scheme remains bounded. It differs from the solution given by other schemes such as the one given in [11] for the case $\rho_{1} / \rho_{2} \rightarrow 1$. This is related to the definition of weak solutions for the system which is not clear from the physical point of view. 


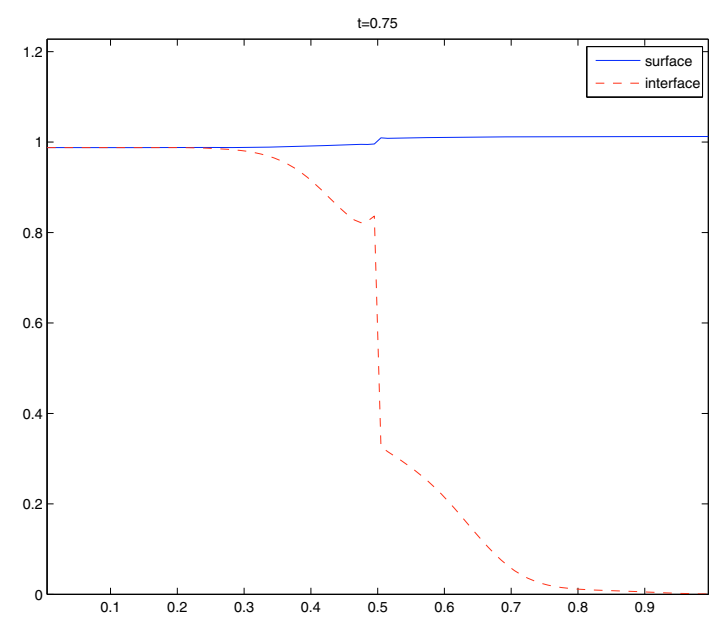

Figure 10. Test 4 with $\rho_{1} / \rho_{2}=0.95$ and kinetic solver.

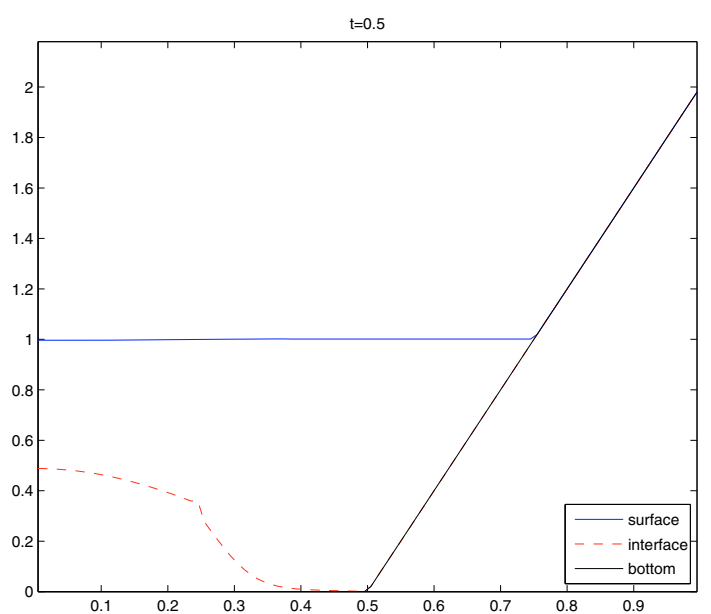

(a)

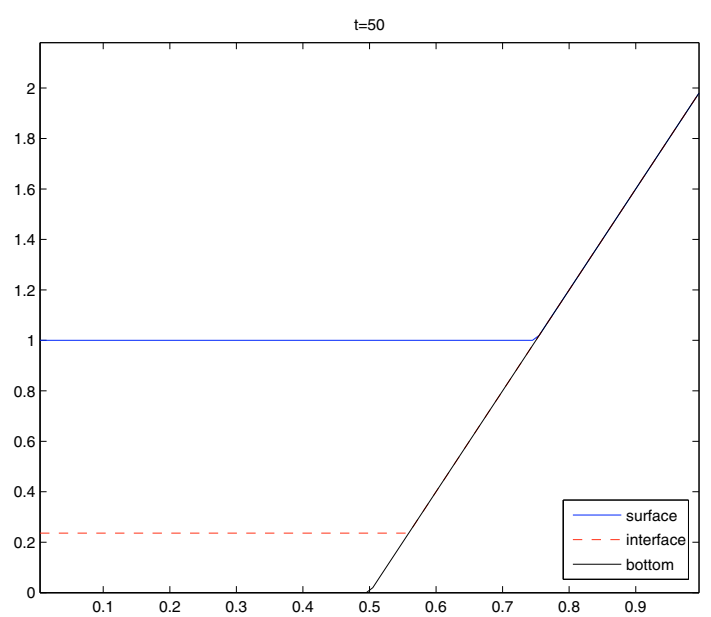

(b)

Figure 11. Test 5 with kinetic solver.

\section{Test 5}

For the last test we take as initial data $g=9.81, \rho_{1} / \rho_{2}=0.95$, and

$$
\begin{aligned}
& h_{1}(t=0, x)=\left(1-h_{2}(t=0, x)-z(x)\right)_{+}, \\
& h_{2}(t=0, x)= \begin{cases}0.5 & \text { if } x<0.25, \\
0 & \text { if } x>0.25,\end{cases} \\
& z(x)= \begin{cases}0 & \text { if } x<0.5, \\
4(x-1 / 2) & \text { if } x>0.5,\end{cases} \\
& u_{1}(t=0, x)=u_{2}(t=0, x)=0 .
\end{aligned}
$$


We solve the system for $x \in[0,1]$ with 100 cells, at CFL 0.5 using the kinetic solver. We impose solid wall boundary conditions on the left, ensuring mass conservation. The result is shown in Figure 11, and we remark again the good behavior in void regions. An hydrostatic steady-state is reached, and is correctly treated by the scheme, according to the well-balanced property.

\section{Conclusion}

When solving the two-layer shallow water system, one can avoid the computation of the eigenvalues of the system by solving each layer separately, and by using some entropy inequality in order to control the stability. Preliminary numerical computations showed that using one-step algorithms that compute each layer independently together with a semi-discrete entropy inequality is not enough in order to have a stable scheme. Thus, we have introduced a time-splitting technique leading to a two-step scheme. We have seen that within this framework, a semi-discrete entropy inequality is not enough anyway to ensure the stability. However, a fully discrete entropy inequality, obtained with the use of the kinetic Saint Venant solver of [17], makes this type of scheme stable. It is then computationally cheap, as the eigenvalues do not need to be computed, uses only the CFL condition of each layer, and does not need special treatment of complex eigenvalues. Moreover, it preserves the nonnegativity of the layers depths, and is well-balanced. The obtained numerical solutions are similar to those given by a global Roe scheme for the two-layer system like in [11], at least when $\rho_{1} / \rho_{2}$ is not too close to 1 . Differences in weak solutions arise between these schemes when $\rho_{1} / \rho_{2} \rightarrow 1$.

\section{REFERENCES}

[1] R. Abgrall and S. Karni, Computations of compressible multifluids. J. Comput. Phys. 169 (2001) 594-623.

[2] R. Abgrall and S. Karni, A relaxation scheme for the two-layer shallow water system, in Proceedings of the 11th International Conference on Hyperbolic Problems (Lyon, 2006), Hyperbolic problems: theory, numerics, applications, S. Benzoni-Gavage and D. Serre Eds., Springer (2007) 135-144.

[3] E. Audusse and M.-O. Bristeau, A well-balanced positivity preserving "second-order" scheme for shallow water flows on unstructured meshes. J. Comput. Phys. 206 (2005) 311-333.

[4] E. Audusse, M.-O. Bristeau and B. Perthame, Kinetic schemes for Saint-Venant equations with source terms on unstructured grids. INRIA Report, RR-3989 (2000).

[5] E. Audusse, F. Bouchut, M.-O. Bristeau, R. Klein and B. Perthame, A fast and stable well-balanced scheme with hydrostatic reconstruction for shallow water flows. SIAM J. Sci. Comput. 25 (2004) 2050-2065 (electronic).

[6] D.S. Bale, R.J. Leveque, S. Mitran and J.A. Rossmanith, A wave propagation method for conservation laws and balance laws with spatially varying flux functions. SIAM J. Sci. Comput. 24 (2002) 955-978 (electronic)

[7] M. Baudin, C. Berthon, F. Coquel, R. Masson and Q.H. Tran, A relaxation method for two-phase flow models with hydrodynamic closure law. Numer. Math. 99 (2005) 411-440.

[8] C. Berthon and F. Coquel, Travelling wave solutions of a convective diffusive system with first and second order terms in nonconservation form, in Hyperbolic problems: theory, numerics, applications, Vol. I (Zürich, 1998), Internat. Ser. Numer. Math. 129, Birkhäuser, Basel (1999) 74-54.

[9] F. Bouchut, Nonlinear stability of finite volume methods for hyperbolic conservation laws and well-balanced schemes for sources, Frontiers in Mathematics. Birkhäuser Verlag, Basel (2004).

[10] F. Bouchut, S. Medvedev, G. Reznik, A. Stegner and V. Zeitlin, Nonlinear dynamics of rotating shallow water: methods and advances, Edited Series on Advances in Nonlinear Science and Complexity. Elsevier (2007).

[11] M. Castro, J. Macías and C. Parés, A $Q$-scheme for a class of systems of coupled conservation laws with source term. Application to a two-layer 1-D shallow water system. ESAIM: M2AN 35 (2001) 107-127.

[12] Q. Jiang and R.B. Smith, Ideal shocks in a 2-layer flow. II: Under a passive layer. Tellus 53A (2001) 146-167.

[13] J.B. Klemp, R. Rotunno and W.C. Skamarock, On the propagation of internal bores. J. Fluid Mech. 331 (1997) 81-106.

[14] M. Li and P.F. Cummins, A note on hydraulic theory of internal bores. Dyn. Atm. Oceans 28 (1998) 1-7.

[15] C. Parés and M. Castro, On the well-balance property of Roe's method for nonconservative hyperbolic systems. Applications to shallow-water systems. ESAIM: M2AN 38 (2004) 821-852.

[16] M. Pelanti, F. Bouchut, A. Mangeney and J.-P. Vilotte, Numerical modeling of two-phase gravitational granular flows with bottom topography, in Proc. of HYP06, Lyon, France (2007).

[17] B. Perthame and C. Simeoni, A kinetic scheme for the Saint-Venant system with a source term. Calcolo 38 (2001) $201-231$.

[18] J.B. Schijf and J.C. Schonfeld, Theoretical considerations on the motion of salt and fresh water, in Proc. of the Minn. Int. Hydraulics Conv., Joint meeting IAHR and Hyd. Div. ASCE (1953) 321-333. 